\title{
Physical Activity and Outcomes in Colorectal Surgery: A Pilot Prospective Cohort Study
}

\author{
David Martin $^{a} \quad$ Benoît Romain $^{b} \quad$ Basile Pache $^{a} \quad$ Aurélie Vuagniaux $^{a}$ \\ Valentine Guarnero ${ }^{a} \quad$ Dieter Hahnloser $^{a} \quad$ Nicolas Demartines $^{a}$ \\ Martin Hübnera \\ aDepartment of Visceral Surgery, University Hospital CHUV and University of Lausanne,

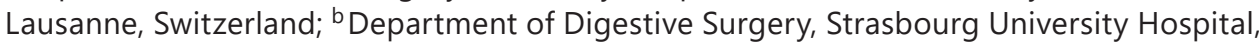 \\ Strasbourg, France
}

\section{Keywords}

Physical activity · Accelerometer · Outcomes · Colorectal surgery

\begin{abstract}
Background: Mobilization after surgery is recommended to reduce the risk of adverse effects and to improve recovery. The aim of this study was to examine the associations between perioperative physical activity and postoperative outcomes in colorectal surgery. Methods: The daily number of footsteps was recorded from preoperative day 5 to postoperative day 3 in a prospective cohort of patients using wrist accelerometers. Timed Up and Go Test (TUGT), 6 Min Walking Test (6MWT), and peak expiratory flow (PEF) were assessed preoperatively. ROC curves were used to assess the performance of physical activity as a diagnostic test of complications and prolonged length of stay (LOS) of more than 5 days. Results: A total of 50 patients were included. Patients with complications were significantly older (67 years) than those without complications (53 years, $p=0.020$ ). PEF was significantly lower in the group with complications (mean flow 294.3 vs. $363.6 \mathrm{~L} / \mathrm{min}, p=0.038$ ) while there was no difference between groups for the other two tests (TUGT and 6MWT). The tests had no capacity to discriminate the occurrence of complications and prolonged LOS, except the 6MWT for LOS (AUC $=0.746$, $p=0.004,95 \% \mathrm{Cl}: 0.604-0.889)$. There was no difference in the mean number of preoperative footsteps, but patients with complications walked significantly less postoperatively (mean daily footsteps 1,101 vs. $1,243, p=0.018$ ). Conclusions: Colorectal surgery patients with complications were elderly, had decreased PEF, and walked less postoperatively. The 6MWT could be used preoperatively to discriminate patients with potentially increased LOS and foster mobilisation strategies.


Martin et al.: Physical Activity and Outcomes in Colorectal Surgery

\section{Introduction}

Patients undergoing colorectal surgery experience a physiologic stress response and are at risk of complications and delayed recovery [1,2]. Attempts to improve recovery have tended to focus on the intraoperative period and the immediate postoperative period (Enhanced Recovery After Surgery, ERAS, pathways) [2]. ERAS reduced overall morbidity, length of hospital stay, and costs by incorporating multimodal optimization of perioperative care first in colorectal surgery and then also in other fields of surgery [3-6].

Early postoperative mobilisation is a fundamental part of the ERAS pathway, as it affects the ability to perform activities of daily living, return to work, and it helps to prevent complications [1]. However, intervention on preoperative factors, such as physical fitness, is an attractive strategy that has developed over the last decade [2]. Few studies have analysed the effect of poor preoperative physical fitness on postoperative recovery, with higher mortality and major complications rate as well as delayed functional recovery after abdominal surgery $[7,8]$. A recent systematic review of randomised trials has demonstrated that prehabilitation combining endurance and resistance training may improve physical fitness as well as quality of life and decrease length of stay (LOS), and postoperative pulmonary complications [9]. However, there are few quantitative data specific to patients and physical activity.

The aim of this pilot prospective study in colorectal surgery was to examine the association between perioperative physical activity and postoperative outcomes.

\section{Materials and Methods}

\section{Patients}

This prospective study included a cohort of non-selected elective colorectal patients (except stoma closure) operated from June 2016 to September 2018. Patients were included at the preoperative surgeon's consultation after clear and appropriate information, with specific consent signed. All patients were treated according to the ERAS protocol including routine control at 30 days after surgery $[10,11]$. Based on clinical considerations, the sample size was set at 50 patients in order to carry out a pilot study at first.

Physical Activity

Timed Up and Go Test (TUGT) and 6 Min Walking Test (6MWT) were chosen to assess functional capacity. TUGT was measured as the time in seconds taken to rise from a chair, walk $3 \mathrm{~m}$, turn, walk back, and sit down again [12]. For 6MWT, patients were told that they had 6 min to walk back and forth along the hallway at their usual pace, and the total distance was recorded in metres using the accelerometer. Reference equations were used to compute the percent predicted 6MWT according to age, gender, height, and weight [13]. Peak expiratory flow (PEF) was measured with a peak flow meter (Clement Clarke ${ }^{\circledR}$; Mini Wright Standard Peak Flow) to monitor patient's ability to breathe out air (maximum speed of expiration in L/min). Reference equations were used to compute the percent predicted PEF according to age, height, and gender [14]. These tests were assessed preoperatively between 1 and 2 weeks prior to surgery through consultation with a dedicated nurse.

Number of daily footsteps was recorded via bracelets containing an accelerometer (E-care Fit ${ }^{\circledR}$ ). Data were synchronized via a tablet on a software and no geolocation was used. The accelerometer was carried on patients from preoperative day 5 to postoperative day 3. Patients were instructed to wear it on the wrist of their dominant hand. Patients who experienced a technical problem with the accelerometer or withdrew their consent during the study were excluded (missing data). One step was defined as measuring $0.75 \mathrm{~m}$.

\section{Postoperative Outcomes}

Postoperative outcomes were prospectively collected until 30 days of surgery. Complications were graded according to the Clavien classification [15]. Major complications were defined as grade IIIb-IVb, and grade $\mathrm{V}$ for mortality, respectively. Only the highest grade was retained in patients presenting more than one complication. In addition, the Comprehensive Complication Index (CCI) was calculated, considering all postoperative complications and thus, giving an estimation of overall morbidity in an individual patient, ranging 
Fig. 1. Study flowchart diagram.

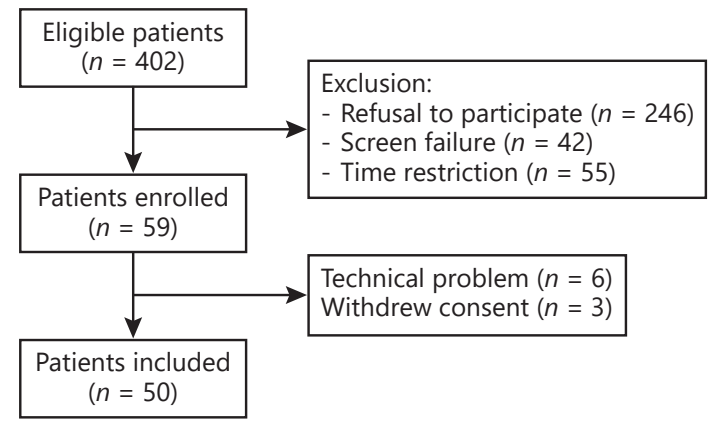

from 0 (no complications) to 100 [16]. LOS was defined as time spent at the hospital from the date of surgery until discharge. Surgical and medical complications were not considered separately.

Outcomes were prospectively recorded by the investigators at 30 days postoperatively which were not aware of walking test data synchronized on the software by a dedicated nurse. Physical tests and accelerometers data were then gathered on a database with outcomes after inclusion of the last patient for analysis. Patients with and without complications were compared in terms of demographics, surgical details, and physical activity.

\section{Statistical Analysis}

Continuous variables were presented as mean (standard deviation, SD) or median (interquartile range, IQR) and compared with Student's t test or Mann-Whitney U test according to their normality. Categorial variables were presented as frequencies (percentage) and compared with Pearson's $\chi^{2}$ or Fisher's exact test as appropriate. Statistical correlation between footsteps and outcomes was measured by use of the Pearson correlation coefficient. Receiver operating characteristic (ROC) curves were used to assess the performance of physical activity as a diagnostic test of complications and prolonged LOS of more than 5 days (defined by the median LOS of the cohort). A good diagnostic performance of a test was defined as a ROC curve having an area under the curve (AUC) of at least 0.7 . Sensitivity and specificity regarding possible cutoff were also calculated. A $p$ value $\leq 0.05$ was considered statistically significant and all tests were two-sided. Analysis was performed using SPSS 22.0 software (SPSS Inc., Chicago, IL, USA).

\section{Results}

A total of 50 colorectal patients were included in this prospective study (Fig. 1). Three patients were excluded because of withdrawal of consent and another 6 patients because of technical problems or non-wearing of the accelerometer. Patient demographics and surgical details are displayed in Table 1. Patients with complications were significantly older (67 years) than those without complications ( 53 years, $p=0.020$ ). Eighteen out of 50 patients had complications (36\%), mostly minor complications (Clavien grade I-IIIa, $n=17$ ), and only 1 patient had a major complication (Clavien grade IVb, 6\%; for all online supplementary material, see www.karger.com/doi/10.1159/000507578). The first complications occurred on mean postoperative day 6 (SD 5.9), the mean CCI of patients with complications was 22.9 and 4 of them had to be readmitted (22\%). The mean LOS was significantly longer in the group of patients with complications ( 8.8 vs. 5 days, $p=0.005$ ).

Physical activity of patients is described in Table 2. In terms of physical tests, PEF was significantly lower in the group of patients with complications (294.3 vs. $363.6 \mathrm{~L} / \mathrm{min}, p=$ 0.038). There was no significant difference between smokers (346 L/min) and non-smokers 
Table 1. Patient demographics and surgical details

\begin{tabular}{lcccc}
\hline & $\begin{array}{l}\text { Overall } \\
(n=50)\end{array}$ & $\begin{array}{l}\text { No complications } \\
(n=32)\end{array}$ & $\begin{array}{l}\text { Complications } \\
(n=18)\end{array}$ & $p$ value* \\
\hline Mean age \pm SD, years & $58.7 \pm 18.2$ & $53.3 \pm 19.9$ & $67.1 \pm 15.4$ & $\mathbf{0 . 0 2 0}$ \\
Mean BMI \pm SD, kg/m ${ }^{2}$ & $25.4 \pm 4.3$ & $25.3 \pm 4.3$ & $25.5 \pm 4.4$ & 0.975 \\
Gender (M: F) & $34: 16$ & $22: 10$ & $11: 7$ & 0.757 \\
Alcohol consumption (>1 unit per day) & $33(66 \%)$ & $24(75 \%)$ & $9(50 \%)$ & 0.212 \\
Active smoking (daily or occasionally) & $19(38 \%)$ & $12(38 \%)$ & $7(39 \%)$ & 0.767 \\
Cardiovascular disease & $19(38 \%)$ & $12(38 \%)$ & $7(39 \%)$ & 0.767 \\
Chronic pulmonary disease & $7(14 \%)$ & $4(13 \%)$ & $3(17 \%)$ & 0.677 \\
Diabetes & $6(12 \%)$ & $2(6 \%)$ & $4(22 \%)$ & 0.162 \\
ASA score (I-II: III-IV) & $43: 7$ & $28: 4$ & $15: 3$ & 0.677 \\
Malignancy & $27(54 \%)$ & $14(44 \%)$ & $13(72 \%)$ & $\mathbf{0 . 0 3 6}$ \\
Surgical procedure & & & & 0.711 \\
$\quad$ Colon & $38(76 \%)$ & $25(78 \%)$ & $3(72 \%)$ & $2(17 \%)$ \\
$\quad$ Rectum & $7(14 \%)$ & $4(13 \%)$ & $14(82 \%)$ & 0.396 \\
Other & $5(10 \%)$ & $3(9 \%)$ & $30(91 \%)$ & \\
\hline
\end{tabular}

SD, standard deviation; BMI, body mass index; ASA, American Society of Anaesthesiologists. * Comparison of patients with $(n=18)$ and without $(n=32)$ complications. Significant $p$ values $(<0.05)$ are displayed in bold characters.

Table 2. Physical activity

\begin{tabular}{|c|c|c|c|c|}
\hline & $\begin{array}{l}\text { Overall } \\
(n=50)\end{array}$ & $\begin{array}{l}\text { No complications } \\
(n=32)\end{array}$ & $\begin{array}{l}\text { Complications } \\
(n=18)\end{array}$ & $p$ value* \\
\hline Mean TUGT \pm SD, s & $9.3 \pm 2.9$ & $9.0 \pm 2.9$ & $9.9 \pm 2.9$ & 0.182 \\
\hline Mean $6 \mathrm{MWT} \pm \mathrm{SD}, \mathrm{m}$ & $422.4 \pm 93.8$ & $440.9 \pm 79.6$ & $389.6 \pm 106.7$ & 0.177 \\
\hline Mean percent predicted $6 \mathrm{MWT} \pm \mathrm{SD}$ & $64 \pm 19$ & $66 \pm 17$ & $59 \pm 19$ & 0.293 \\
\hline Mean $\mathrm{PEF} \pm \mathrm{SD}, \mathrm{L} / \mathrm{min}$ & $340.1 \pm 102.1$ & $363.6 \pm 99.6$ & $294.3 \pm 90.9$ & 0.038 \\
\hline Mean percent predicted $\mathrm{PEF} \pm \mathrm{SD}$ & $73 \pm 19$ & $75 \pm 19.8$ & $71 \pm 17.9$ & 0.436 \\
\hline Mean of daily preoperative footsteps \pm SD, $n$ & $6,444 \pm 4,095$ & $6,384 \pm 4,09$ & $6,573 \pm 4,098$ & 0.752 \\
\hline Mean of daily postoperative footsteps \pm SD, $n$ & $1,191 \pm 1,864$ & $1,243 \pm 1,641$ & $1,101 \pm 2,198$ & 0.018 \\
\hline
\end{tabular}

TUGT, Timed Up and Go Test; 6MWT, 6 Min Walking Test; PEF, peak expiratory flow. ${ }^{*}$ Comparison of patients with $(n=18)$ and without $(n=32)$ complications. Significant $p$ values $(<0.05)$ are displayed in bold characters. The mean of daily footsteps of residents in surgery was 9,587 $\pm 4,450$.

(336 L/min, $p=0.825$ ). The mean number of daily footsteps according to the presence of complications is displayed in Figure 2. No significant difference was found between the number of steps and the presence of complications at any of the 5 preoperative and 3 postoperative days. There was no difference in terms of overall preoperative footsteps between the two groups. However, the mean number of overall daily postoperative footsteps was significantly lower in patients with complications compared to those without complications $(1,101$ vs. $1,243, p=0.018)$, which represents in distance 932 versus $826 \mathrm{~m}$. Patients without complications walked on average $557 \mathrm{~m}$ on operative day, 1,023 m on day 1,1,152 m on day 2 , and $1,008 \mathrm{~m}$ on day 3 . Postoperative/preoperative footsteps ratio was $19.5 \%$ in patients without complications and $16.5 \%$ in patients with complications $(p=0.038)$. 


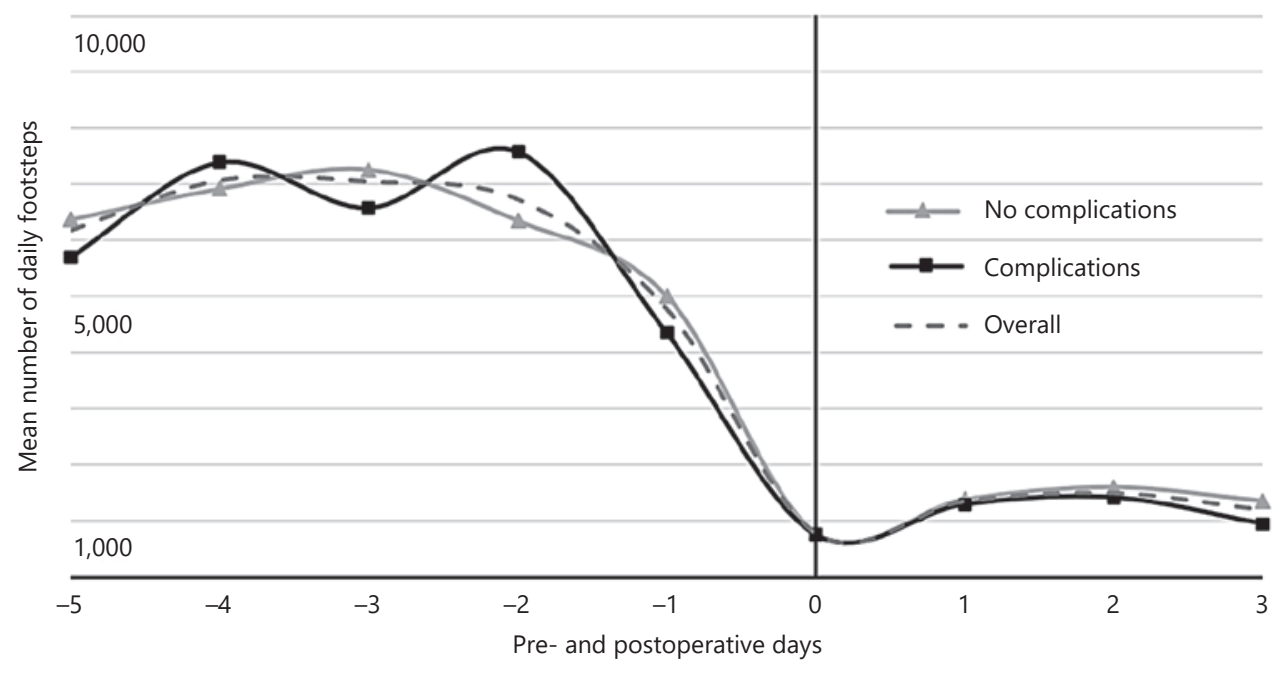

Fig. 2. Physical activity according to complications.

Fig. 3. Correlation between post-

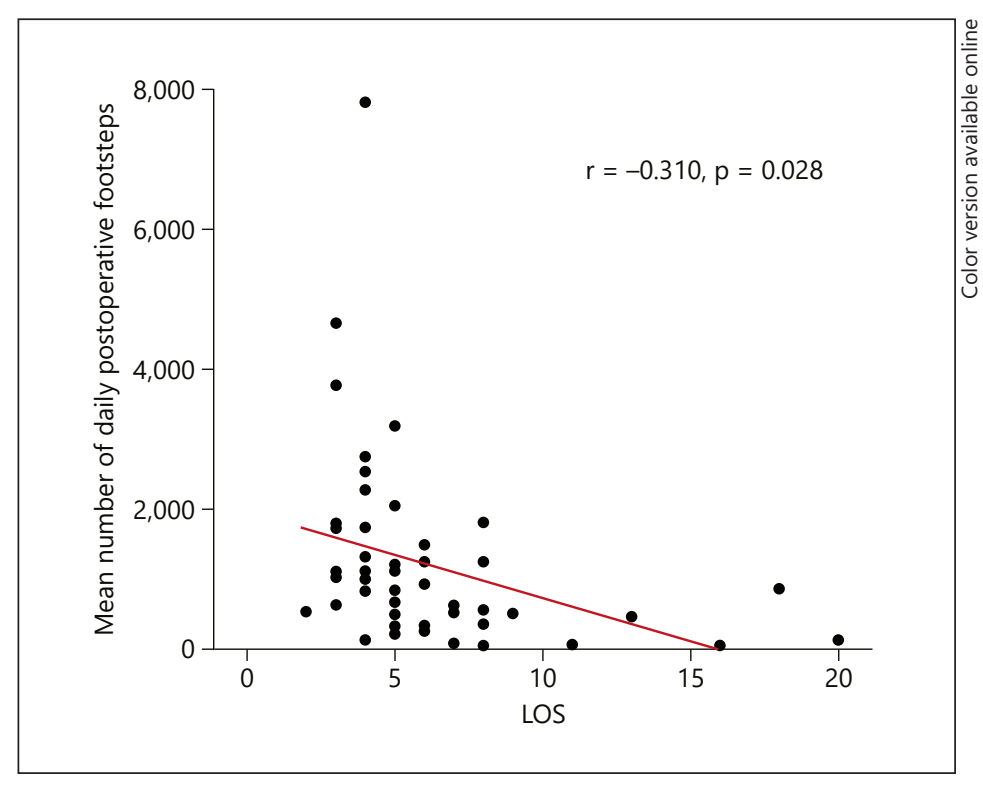
operative footsteps and LOS.

No correlation was found between the number of pre- or postoperative footsteps and the $\mathrm{CCI}$, as well as the number of preoperative footsteps and LOS. On the other hand, there was a significant negative relationship between number of postoperative footsteps and LOS ( $\mathrm{r}=$ $-0.310, p=0.028$; Fig. 3). No correlation was found between 6MWT, TUGT, PEF, and complications or LOS.

Physical tests had no diagnostic ability to assess complications (Fig. 4a), but the 6MWT showed significant ability to discriminate prolonged LOS of more than 5 days (AUC $=0.746$, $p=0.004,95 \%$ CI: 0.604-0.889; Fig. 4b). A cutoff distance of $502 \mathrm{~m}$ had a true positive (sensitivity) of 0.31 , a specificity of 0.95 (false positive rate of 0.05 ), and a positive predictive value of 0.79 to predict prolonged LOS. 


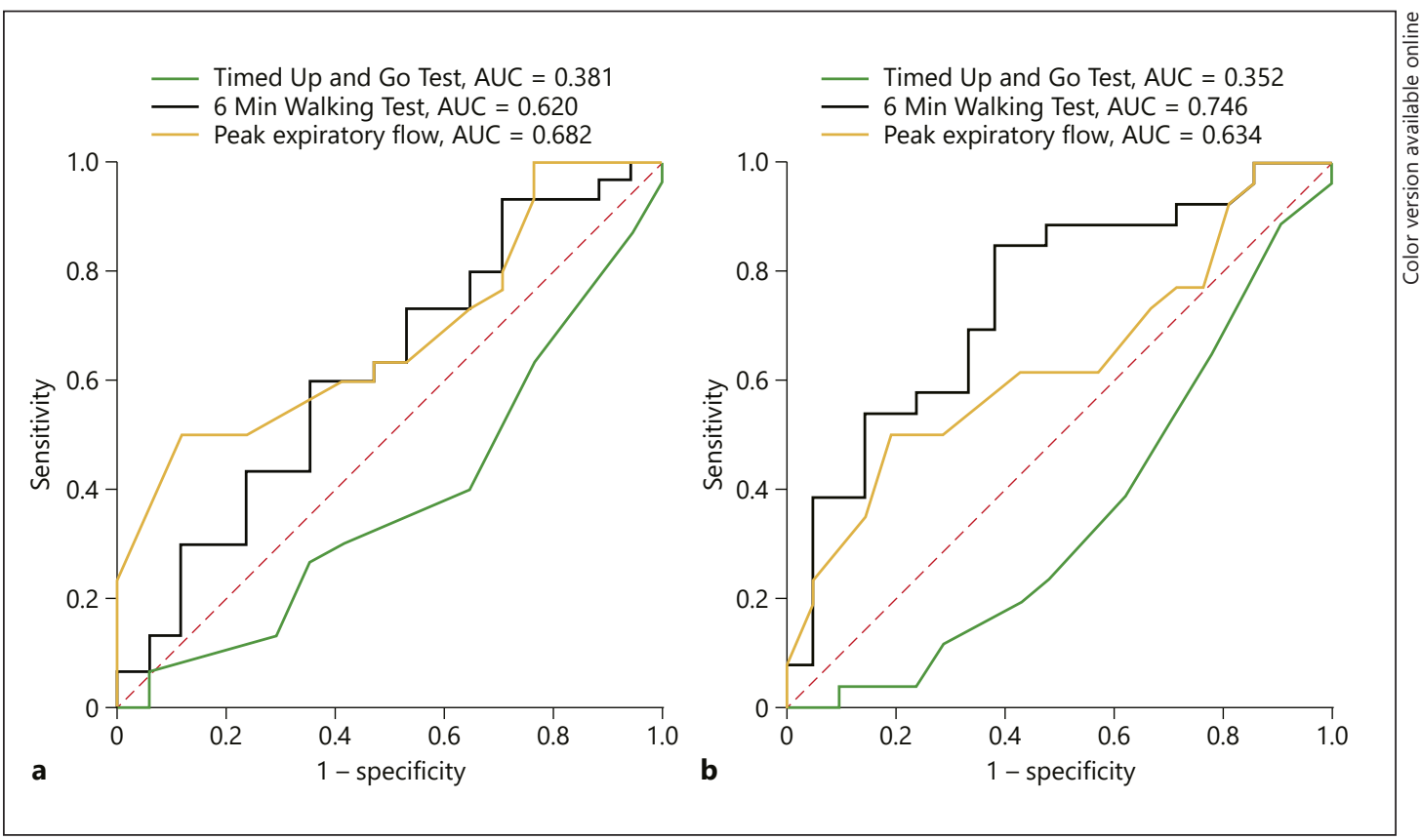

Fig. 4. a ROC curve of physical tests and complications. b ROC curve of physical tests and prolonged LOS.

\section{Discussion}

This prospective cohort study using accelerometers allowed to quantify physical activity in the perioperative course of colorectal patients. The mean number of overall daily postoperative footsteps was significantly lower in patients with complications, and a significant correlation was found between number of postoperative footsteps and LOS. Moreover, the 6MWT showed significant ability to discriminate prolonged LOS.

In the present study, $36 \%$ of patients had complications, mostly minor, and the mean CCI in patients with complications was 22.9 , which is comparable to other cohorts of colorectal surgery $[17,18]$. The LOS was, as expected, higher in the group of patients with complications. No correlation was found between the number of pre- or postoperative footsteps and the CCI, as well as the number of preoperative footsteps and LOS. Furthermore, there was no difference in terms of overall preoperative footsteps between the patients with and without complications. Similarly, a prospective observational study of 327 colorectal patients showed the absence of association between physical activity level before surgery and postoperative recovery [19]. In contrast to these results, one study concluded that a higher preoperative physical activity level was associated with faster self-reported recovery after surgery [20]. However, in the present study, the mean number of overall daily postoperative footsteps was significantly lower in patients with complications compared to those without complications, and a significant negative relationship between number of postoperative footsteps and LOS was observed. One possible explanation is that patients with complications were more bedridden and walked less. Nevertheless, complications occurred on average on the 6th postoperative day and may not have influenced the ability to walk postoperatively (days $0-3$ ). Early postoperative activity is probably thus not only a predictor but also a causative factor for the risk of complications and the length of hospital stay [1]. This cause-effect relationship cannot, however, be confirmed by the present study since the recording of steps was limited to the first 3 postoperative days and complications occurred on the 6th postoperative day. A 
retrospective cohort study on colorectal patients showed that more than half of the patients did not mobilise as recommended by the ERAS guidelines, and delayed mobilisation was associated with increased postoperative complications [21]. Low physical functioning is associated with disability and a loss of independence, and not all individual colorectal cancer patients recover to their preoperative level of physical functioning [19]. In the present study, the drop in the number of postoperative footsteps compared to the preoperative footsteps was significantly higher in patients with complications (83.5 vs. $80.5 \%$ ). Nowadays, as the vast majority of smartphones measure the number of steps, this represents an interesting tool, and cutoffs could be used in the future to set postoperative goals for patients.

Patients with postoperative complications were significantly older than those without complications. These results could reflect the effect of aging on physical fitness. However, in a precedent pilot study, physical activity increased steadily in the postoperative course regardless of age [1]. On the other side, increasing patient age at the time of surgical resection of colorectal cancer is associated with an increase in both morbidity and mortality [22, 23]. The advent of laparoscopy in colorectal surgery, widely used in this cohort $(>80 \%)$, has substantially reduced morbidity and LOS and improved quality of life [24-26]. This minimally invasive approach is also safe and feasible in elderly patients [27]. Older patients were also more susceptible to infective complications postoperatively in a prospective study of 163 consecutive patients undergoing laparoscopic colorectal surgery [28]. Another retrospective study including colorectal patients in an ERAS pathway showed that despite more comorbidities, older patients did not experience more complications [29]. Furthermore, minimally invasive surgery and high compliance with the ERAS protocol can help to prevent postoperative pulmonary complications in the elderly [30]. Regarding the oncological aspect of the present cohort, the malignancy rate was higher in patients with complications. These results could also reflect the effect of cancer. In view of the small sample, no conclusion can be drawn on this aspect. On the other hand, several studies showed that physical activity was associated with better quality of life and extended survival after cancer diagnosis [31-33]. A systematic review also confirmed that physical activity was associated with improved survival in an oncological population [33].

Objective measures were already used to show that moderate- to vigorous-intensity physical activity (MVPA) using an accelerometer was associated with better health-related quality of life (HRQoL), physical function, and well-being [34]. Another cross-sectional study of 1,371 colorectal cancer patients confirmed that MVPA was positively associated with physical HRQoL [35]. Physical activity was also associated with lower fatigue, pain, and insomnia [36]. In 1,996 colorectal cancer survivors interviewed by phone, self-measured physical activity had a positive association with the physical and functional well-being [37].

Outside of footsteps, a better preoperative physical performance assessed with the TUGT or walking distance was associated with reduced rate of complications after colorectal cancer surgery $[17,18,38]$. In the present study, the 6MWT showed significant ability to discriminate prolonged LOS, with a mean distance of $502 \mathrm{~m}$ as diagnostic threshold. This is in line with another prospective study of 191 colorectal patients which showed that increased walking distance (mean 6MWT of $462 \mathrm{~m}$ ) was associated with fewer complications and a lower rate of discharge to a geriatric care facility or residential care/nursing home, rather than home [39]. Preoperative lack of physical fitness appears to be a risk factor for poorer postoperative outcomes; however, this could not be confirmed by the present study, since 6MWT and preoperative footsteps were not significantly different in the cohort of patients with complications. Moreover, a Canadian study demonstrated that slower walking was associated with increased postoperative morbidity after elective colorectal resection [18]. Otherwise, 6MWT of less than $325 \mathrm{~m}$ has been described as threshold for predicting postoperative pulmonary complications with $77 \%$ sensitivity and $100 \%$ specificity in patients

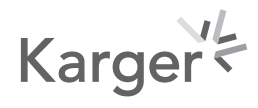


undergoing elective surgery under general anaesthesia [40]. Regarding TUGT, a prospective cohort study of 272 patients including colorectal surgery showed that a score of $15 \mathrm{~s}$ or longer was associated with a significantly increased occurrence of postoperative complications, 30-day readmission, and 1-year mortality [38]. Moreover, the authors demonstrated that slower scores were associated with increased postoperative complications: fast $(\leq 10 \mathrm{~s})$ : 13\%; intermediate (11-14 s): 29\%; and slow ( $\geq 15$ s): 77\% [38]. This is opposed to the present cohort where patients tended to have good scores (mean TUGT $9.3 \mathrm{~s}$ ), which could also be explained by the high proportion of ASA I-II score (86\%). On the other hand, there is actually no single TUGT score that is accepted as a standard cut point for poor performance.

On the respiratory level, this study identified that the absolute PEF value was significantly lowered in the group of patients with complications, but not when compared with the theoretical value depending on the patients' age, size, and gender. Interestingly, there was no difference between smokers and non-smokers. It has already been shown that higher preoperative maximal inspiratory pressure was significantly associated with shorter LOS in colorectal surgery [41, 39]. Furthermore, a randomized study showed that pulmonary function was better preserved after laparoscopic colorectal resection (mean PEF $216 \mathrm{~L} / \mathrm{min}$ ) with consequent decreased pulmonary complications [42].

Feasibility of a prospective study is based on recruitment and compliance to the intervention [43]. In the present study, the patient recruitment rate was low (15\%), as a large majority refused to participate due to lack of interest. The clear reasons for refusals were not formally collected in this study. Problems related to the organization of recruitment on the surgeon's side were also noted (lack of time and organization). However, although the sample size was limited, once the study has been accepted by the patients, the overall acceptability of the intervention was good, with $85 \%$ of them having completed the study (15\% did not due to technical problems related to the bracelet or withdrawal of consent). As described previously in another colorectal surgery cohort, patient engagement represented a major obstacle for the success of a prospective study assessing physical activity [43]. The present study should be considered as pilot and has highlighted the logistical issues and the challenges in recruiting patients to assess their physical activity. Additional prospective investigations are required to validate correlations between physical activity and outcomes specifically in colorectal surgery.

Several limitations of the present study need to be addressed. The device used was an entry-level model that only measured daily footsteps, thus preventing any additional measures, such as minutes spent in light, moderate, and vigorous activity, but also sedentary time. Moreover, there is no literature on its accuracy and precision. The algorithms to count steps could be notoriously flawed, and the numbers may therefore be way off. Validity varied widely between commercially available devices. For example, in a validation study, the Apple Watch was the most accurate for step count in free-living conditions, while other models (iHealth Edge, Misfit Shine) underestimate or overestimate the number of step [44]. Another study found that four different wrist-worn monitors could not adequately measure the number of steps in a population of older adults using rollators [45]. Also, setting the step length to $75 \mathrm{~cm}$ has certainly introduced a bias, especially in the postoperative phase, where step length might have dropped. For practical reasons, the 6MWT test was measured using the accelerometer, and not by the distance covered in the corridor, which could also introduce biases. Otherwise, the patient recruitment rate $(15 \%)$ was low. One hypothesis is that patients had to constantly wear their accelerometer on the dominant hand, which may be uncomfortable for some of them in their daily activities. Another hypothesis is that patients may be scared to be followed in their movements, even if it was not geolocated. Potential systematic errors arising from selection bias were possible, whereas more health-conscious individuals tend to participate in studies involving measurements of physical fitness. An overestimation of the number of steps was also possible, since a patient who became aware of having an accelerometer might be 
walking more. Otherwise, due to the lack of preliminary data and available studies, the sample size was estimated based on clinical considerations, which explains its small size. Furthermore, the number of events may have obscured potential predictors of poor outcomes. Finally, given the underpowered sample, some potential correlations and subtypes of complications were not analysed, such as the occurrence of pulmonary complications based on PEF, for example, or age and malignancy which were not taken as co-factors in the various analysis. There may not be a strong link between physical activity and grade I complications, like urinary retention or superficial infection, but rather to cardiorespiratory complications which were not prevalent in this cohort (6\%). Thus, the relationship with walking fitness and complications may not be linear and not to all complications.

\section{Conclusions}

Colorectal surgery patients with complications were elderly, had decreased PEF, and walked less postoperatively. Objectively measured physical performance is an important factor in perioperative risk assessment, and 6MWT could be used preoperatively to discriminate patients with potentially increased LOS and in a need of a prehabilitation strategy and fostered postoperative mobilisation strategies.

\section{Statement of Ethics}

This study was approved by the Cantonal Ethics Commission (CER-VD protocol number 383/15) and registered under clinicaltrials.gov (NCT02610790).

\section{Disclosure Statement}

The authors have no conflicts of interest to declare.

\section{Funding Sources}

No funding was received.

\section{Author Contributions}

Study conception and design: D.M., B.R., and M.H. Acquisition of data: D.M., B.R., B.P., A.V., and V.G. Analysis and interpretation of data: D.M., B.R., and M.H. Drafting of the manuscript: D.M., B.R., B.P., A.V., V.G., D.H., N.D., and M.H. Critical revision of the manuscript: D.M., B.R., B.P., A.V., V.G., D.H., N.D., and M.H.

\section{References}

1 Romain B, Hübner M, Brigand C, Steimetz JP, Rohr S. Postoperative Footsteps and Outcomes - A Pilot Study. Gen Med (Los Angel). 2016;(4):239. DOI:10.4172/2327-5146.1000239

2 Li C, Carli F, Lee L, Charlebois P, Stein B, Liberman AS, et al. Impact of a trimodal prehabilitation program on functional recovery after colorectal cancer surgery: a pilot study. Surg Endosc. 2013 Apr;27(4):1072-82.

3 Roulin D, Donadini A, Gander S, Griesser AC, Blanc C, Hübner M, et al. Cost-effectiveness of the implementation of an enhanced recovery protocol for colorectal surgery. Br J Surg. 2013 Jul;100(8):1108-14.

4 Greco M, Capretti G, Beretta L, Gemma M, Pecorelli N, Braga M. Enhanced recovery program in colorectal surgery: a meta-analysis of randomized controlled trials. World J Surg. 2014 Jun;38(6):1531-41.

5 Bond-Smith G, Belgaumkar AP, Davidson BR, Gurusamy KS. Enhanced recovery protocols for major upper gastrointestinal, liver and pancreatic surgery. Cochrane Database Syst Rev. 2016 Feb;2:CD011382. 
6 Thanh NX, Chuck AW, Wasylak T, Lawrence J, Faris P, Ljungqvist O, et al. An economic evaluation of the Enhanced Recovery After Surgery (ERAS) multisite implementation program for colorectal surgery in Alberta. Can J Surg. 2016 Dec;59(6):415-21.

7 Lawrence VA, Hazuda HP, Cornell JE, Pederson T, Bradshaw PT, Mulrow CD, et al. Functional independence after major abdominal surgery in the elderly. J Am Coll Surg. 2004 Nov;199(5):762-72.

8 Wilson RJ, Davies S, Yates D, Redman J, Stone M. Impaired functional capacity is associated with all-cause mortality after major elective intra-abdominal surgery. Br J Anaesth. 2010 Sep;105(3):297-303.

9 Piraux E, Caty G, Reychler G. Effects of preoperative combined aerobic and resistance exercise training in cancer patients undergoing tumour resection surgery: A systematic review of randomised trials. Surg Oncol. 2018 Sep;27(3):584-94.

10 Gustafsson UO, Scott MJ, Schwenk W, Demartines N, Roulin D, Francis N, et al.; Enhanced Recovery After Surgery (ERAS) Society, for Perioperative Care; European Society for Clinical Nutrition and Metabolism (ESPEN); International Association for Surgical Metabolism and Nutrition (IASMEN). Guidelines for perioperative care in elective colonic surgery: Enhanced Recovery After Surgery (ERAS( ${ }^{\circledR}$ )) Society recommendations. World J Surg. 2013 Feb;37(2):259-84.

11 Nygren J, Thacker J, Carli F, Fearon KC, Norderval S, Lobo DN, et al.; Enhanced Recovery After Surgery (ERAS) Society, for Perioperative Care; European Society for Clinical Nutrition and Metabolism (ESPEN); International Association for Surgical Metabolism and Nutrition (IASMEN). Guidelines for perioperative care in elective rectal/pelvic surgery: Enhanced Recovery After Surgery (ERAS $\left({ }^{\circledR}\right)$ ) Society recommendations. World J Surg. 2013 Feb;37(2):285-305.

12 Podsiadlo D, Richardson S. The timed "Up \& Go": a test of basic functional mobility for frail elderly persons. J Am Geriatr Soc. 1991 Feb;39(2):142-8.

13 Enright PL, Sherrill DL. Reference equations for the six-minute walk in healthy adults. Am J Respir Crit Care Med. 1998 Nov;158(5 Pt 1):1384-7.

14 Hankinson JL, Odencrantz JR, Fedan KB. Spirometric reference values from a sample of the general U.S. population. Am J Respir Crit Care Med. 1999 Jan;159(1):179-87.

15 Dindo D, Demartines N, Clavien PA. Classification of surgical complications: a new proposal with evaluation in a cohort of 6336 patients and results of a survey. Ann Surg. 2004 Aug;240(2):205-13.

16 Slankamenac K, Graf R, Barkun J, Puhan MA, Clavien PA. The comprehensive complication index: a novel continuous scale to measure surgical morbidity. Ann Surg. 2013 Jul;258(1):1-7.

17 Nutt CL, Russell JC. Use of the pre-operative shuttle walk test to predict morbidity and mortality after elective major colorectal surgery. Anaesthesia. 2012 Aug;67(8):839-49.

18 Lee L, Schwartzman K, Carli F, Zavorsky GS, Li C, Charlebois P, et al. The association of the distance walked in 6 min with pre-operative peak oxygen consumption and complications 1 month after colorectal resection. Anaesthesia. 2013 Aug;68(8):811-6.

19 van Zutphen M, Winkels RM, van Duijnhoven FJ, van Harten-Gerritsen SA, Kok DE, van Duijvendijk P, et al. An increase in physical activity after colorectal cancer surgery is associated with improved recovery of physical functioning: a prospective cohort study. BMC Cancer. 2017 Jan;17(1):74.

20 Onerup A, Bock D, Börjesson M, Fagevik Olsén M, Gellerstedt M, Haglind E, et al. Is preoperative physical activity related to post-surgery recovery?-a cohort study of colorectal cancer patients. Int J Colorectal Dis. 2016 Jun;31(6):1131-40.

21 Grass F, Pache B, Martin D, Addor V, Hahnloser D, Demartines N, et al. Feasibility of early postoperative mobilisation after colorectal surgery: A retrospective cohort study. Int J Surg. 2018 Aug;56:161-66.

22 Damhuis RA, Wereldsma JC, Wiggers T. The influence of age on resection rates and postoperative mortality in 6457 patients with colorectal cancer. Int J Colorectal Dis. 1996;11(1):45-8.

23 Simmonds PD, Best L, George S, Baughan C, Buchanan R, Davis C, et al.; Colorectal Cancer Collaborative Group. Surgery for colorectal cancer in elderly patients: a systematic review. Lancet. 2000 Sep;356(9234):968-74.

24 Weeks JC, Nelson H, Gelber S, Sargent D, Schroeder G; Clinical Outcomes of Surgical Therapy (COST) Study Group. Short-term quality-of-life outcomes following laparoscopic-assisted colectomy vs open colectomy for colon cancer: a randomized trial. JAMA. 2002 Jan;287(3):321-8.

25 Veldkamp R, Kuhry E, Hop WC, Jeekel J, Kazemier G, Bonjer HJ, et al.; COlon cancer Laparoscopic or Open Resection Study Group (COLOR). Laparoscopic surgery versus open surgery for colon cancer: short-term outcomes of a randomised trial. Lancet Oncol. 2005 Jul;6(7):477-84.

26 Green BL, Marshall HC, Collinson F, Quirke P, Guillou P, Jayne DG, et al. Long-term follow-up of the Medical Research Council CLASICC trial of conventional versus laparoscopically assisted resection in colorectal cancer. Br J Surg. 2013 Jan;100(1):75-82.

27 Luglio G, Nelson H. Laparoscopy for colon cancer: state of the art. Surg Oncol Clin N Am. 2010 Oct;19(4):77791.

28 Kvasnovsky CL, Adams K, Sideris M, Laycock J, Haji AK, Haq A, et al. Elderly patients have more infectious complications following laparoscopic colorectal cancer surgery. Colorectal Dis. 2016 Jan;18(1):94-100. https://doi.org/10.1111/codi.13109.

29 Slieker J, Frauche P, Jurt J, Addor V, Blanc C, Demartines N, et al. Enhanced recovery ERAS for elderly: a safe and beneficial pathway in colorectal surgery. Int J Colorectal Dis. 2017 Feb;32(2):215-21.

30 Jurt J, Hübner M, Pache B, Hahnloser D, Demartines N, Grass F. Respiratory Complications After Colorectal Surgery: avoidable or Fate? World J Surg. 2018 Sep;42(9):2708-14.

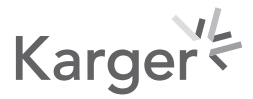


Martin et al.: Physical Activity and Outcomes in Colorectal Surgery

31 Fong DY, Ho JW, Hui BP, Lee AM, Macfarlane DJ, Leung SS, et al. Physical activity for cancer survivors: metaanalysis of randomised controlled trials. BMJ. 2012 Jan;344 jan30 5:e70.

32 Gorzelitz J, Costanzo ES, Spencer RJ, Rumble M, Rose SL, Cadmus-Bertram L. Longitudinal assessment of postsurgical physical activity in endometrial and ovarian cancer patients. PLoS One. 2019 Oct;14(10):e0223791.

33 McTiernan A, Friedenreich CM, Katzmarzyk PT, Powell KE, Macko R, Buchner D, et al.; 2018 PHYSICAL ACTIVITY GUIDELINES ADVISORY COMMITTEE*. Physical Activity in Cancer Prevention and Survival: A Systematic Review. Med Sci Sports Exerc. 2019 Jun;51(6):1252-61.

34 Vallance JK, Boyle T, Courneya KS, Lynch BM. Associations of objectively assessed physical activity and sedentary time with health-related quality of life among colon cancer survivors. Cancer. 2014 Sep;120(18): 2919-26.

35 Buffart LM, Thong MS, Schep G, Chinapaw MJ, Brug J, van de Poll-Franse LV. Self-reported physical activity: its correlates and relationship with health-related quality of life in a large cohort of colorectal cancer survivors. PLoS One. 2012;7(5):e36164.

36 Grimmett C, Bridgewater J, Steptoe A, Wardle J. Lifestyle and quality of life in colorectal cancer survivors. Qual Life Res. 2011 Oct;20(8):1237-45.

37 Lynch BM, Cerin E, Owen N, Aitken JF. Associations of leisure-time physical activity with quality of life in a large, population-based sample of colorectal cancer survivors. Cancer Causes Control. 2007 Sep;18(7):73542.

38 Robinson TN, Wu DS, Sauaia A, Dunn CL, Stevens-Lapsley JE, Moss M, et al. Slower walking speed forecasts increased postoperative morbidity and 1-year mortality across surgical specialties. Ann Surg. 2013 Oct; 258(4):582-8; discussion 88-90.

39 Karlsson E, Egenvall M, Farahnak P, Bergenmar M, Nygren-Bonnier M, Franzen E, et al. Better preoperative physical performance reduces the odds of complication severity and discharge to care facility after abdominal cancer resection in people over the age of 70 - A prospective cohort study. Eur J Surg Oncol. 2018 Nov; 44(11): 1760-67.

40 Sinclair RC, Batterham AM, Davies S, Cawthorn L, Danjoux GR. Validity of the 6 min walk test in prediction of the anaerobic threshold before major non-cardiac surgery. Br J Anaesth. 2012 Jan;108(1):30-5.

41 Dronkers JJ, Chorus AM, van Meeteren NL, Hopman-Rock M. The association of pre-operative physical fitness and physical activity with outcome after scheduled major abdominal surgery. Anaesthesia. 2013 Jan;68(1): 67-73.

42 Schwenk W, Bohm B, Witt C, Junghans T, Grundel K, Muller JM. Pulmonary function following laparoscopic or conventional colorectal resection: a randomized controlled evaluation. Arch Surg. 1999 Jan;134(1):6-12; discussion 13. https://doi.org/10.1001/archsurg.134.1.6.

43 Northgraves MJ, Arunachalam L, Madden LA, Marshall P, Hartley JE, MacFie J, et al. Feasibility of a novel exercise prehabilitation programme in patients scheduled for elective colorectal surgery: a feasibility randomised controlled trial. Supportive Care in Cancer. 2019 Nov. https://doi.org/10.1007/s00520-01905098-0.

44 Breteler MJ, Janssen JH, Spiering W, Kalkman CJ, van Solinge WW, Dohmen DA. Measuring Free-Living Physical Activity With Three Commercially Available Activity Monitors for Telemonitoring Purposes: Validation Study. JMIR Form Res. 2019 Apr 24;3(2):e11489.

45 Larsen RT, Korfitsen CB, Juhl CB, Andersen HB, Langberg H, Christensen J. Criterion validity for step counting in four consumer-grade physical activity monitors among older adults with and without rollators. Eur Rev Aging Phys Act. 2020 Jan;17:1. https://doi.org/10.1186/s11556-019-0235-0. 